\title{
Application of FDEMS for geological horizons mapping
}

\author{
I.Ingerov* \\ Advanced Geophysical Operations and Services Inc. (AGCOS)
}

\author{
A. Lozovoy ,Y. Mendriy \\ National Mining University of Ukrain
}

\begin{abstract}
The frequency domain electromagnetic methods (FDEM) could be separated in two groups: 1)Shallow FDEM profiling techniques which use high frequency bands and mainly utilize only the magnetic components of the EM field; 2)Electromagnetic soundings in wide depths intervals, consisting of two sub methods: the impedance soundings (CSAMT); and the individual components EM soundings (FDEMS); the last sub method combines geometric and induction soundings techniques. Both sub methods of electromagnetic soundings actively use magnetic and electrical EM field components of the control source and, whereas in CSAMT only the far zone of the control source is being used, FDEMS engages all three control source zones: nearest, middle and far. Such feature provides a number of significant advantages during interpretation of the field data. At the present time, the hardware capabilities of recently developed wideband $(50000-0.0001 \mathrm{~Hz}$ ) multifunction EM receivers GEPARD-4 and GEPARD-8 allow to take full advantage from high resolution FDEMS method application for solving a variety geological engineering and groundwater exploration tasks in a wide range of depths $(2 \mathrm{~m}$ to $5 \mathrm{~km})$.
\end{abstract}

Keywords—fdems; geological horizons; mapping; em

\section{INTRODUCTION}

The frequency domain electromagnetic (FDEM) methods could be separated into two groups:1) Shallow FDEM profiling techniques which use high frequency bands and mainly utilize only the magnetic components of the EM field $^{[1]}$;2)Electromagnetic soundings in wide depth intervals, consisting of two sub methods: the impedance soundings (CSAMT); and the individual components EM soundings (FDEMS); the last sub method combines geometric and induction soundings techniques.

The CSAMT method is well known and widely used all over the world. It was introduced in 1980s of the last century as a method which allowed to extend the range of magnetotelluric measurements to higher frequencies ${ }^{[2]}$. In CSAMT, the grounded electric dipole (AB) is commonly used as the control source of the EM field and measurements of the source's electric component EX and orthogonal to it horizontal magnetic component $\mathrm{Hy}$ are carried out in the far zone of the control source's equatorial area. In this case, apparent resistivity ( $\left.\rho_{x y}\right)$ is calculated similar to the MT method, by normalizing the electric field to the orthogonal magnetic field $^{[3]}$. A more comprehensive version of the high frequency magnetotelluric field with two orthogonal horizontal magnetic dipoles as the source of EM field was also proposed by geophysical equipment manufacturers.

Practical implementation of the frequency domain soundings (FDEMS) method, which is based on the discrete application of five EM field components, was for the most part unnoticed around the world. The FDEMS method was developed in 1950s of the last century and was actively used during various field surveys from 1950s to 1990s in the former $\mathrm{USSR}^{[4]}$. During this period of time, the Geological Institute of Academy of Sciences of USSR, in conjunction with the development of data interpretation methods, designed several sets of field equipment: low $(100-0.01 \mathrm{~Hz})$, medium $(2000-1$ $\mathrm{Hz})$ and high frequency $(10000-70 \mathrm{~Hz})^{[5]}$. The super high

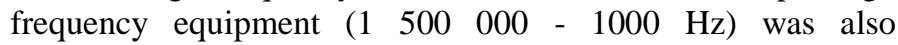
developed for geological engineering surveys mainly for the construction industry applications. The development of interpretation methods was focused primarily on the analysis of coordinates of the characteristic points (extremes) due to the limited capabilities of the computer technologies which existed at the time $\mathrm{e}^{[5-7]}$. The high accuracy of mapping the surface of the high resistivity reference horizons with FDEMS method is attributed to the presence of elements on its curves, which are closely related to the ratio of soundings spacing (R) and the depth $(\mathrm{H})$ of the high resistivity reference horizon ${ }^{[6,7]}$. At the present time, this unique feature of the method could be applied in the field with greater effectiveness by exploiting the hardware and software capabilities of modern EM instruments. The most suitable equipment set for the implementation of FDEMS method in the field is the wideband multifunction data acquisition system recently developed in Canada, consisting of 8- channel EM receiver GEPARD-8 (equipped with flexible configuration of electric and magnetic channels), AT-100 transmitter, magnetic sensors AMS-15 and AMS-37, and precision field tripods for quick and accurate magnetic sensors installation on any terrain or soil conditions ${ }^{[8]}$. The availability of such EM equipment created he perfect conditions for the practical application of all FDEMS method advantages for mining exploration, mapping and solving a variety of geological and engineering problems.

\section{Methodology OF FIELD Work With Fdems Method}

For the FDEMS surveys, typically the grounded electric dipole is used as a source. Measurements for the horizontal electrical component $\left(E_{x}{ }^{y}\right)$, which is parallel to the source dipole and frequency derivate of the vertical magnetic component $\left(\partial \mathrm{B}_{\mathrm{z}}^{\mathrm{y}} / \partial \omega\right)$ are carried out in the equatorial area of the source, with the sounding spacing (R) being 2-10 times greater than the depth $(\mathrm{H})$ of investigation. These two components of the EM field are the most sensitive to the changes of geoelectrical cross-section parameters ${ }^{[7,8]}$. From the field survey productivity standpoint, quite often just the horizontal axial electrical component $\left(\mathrm{E}_{\mathrm{x}}{ }^{\mathrm{x}}\right)$ is measured. For all the instances, at every observation site measured are the 
amplitude of the signal and its differential phase parameter, defined as normalized by frequency phase shift between signal's $1^{\text {st }}$ and $3^{\text {rd }}$ harmonics $(\Delta \varphi \omega)$. The physical meaning of this parameter is described by the equation (1) as the sum of signal's phase minus its frequency derivative normalized to frequency ${ }^{[9]}$ :

$$
(\Delta \varphi \omega)=\varphi \omega-\omega \times \partial \varphi \omega / \partial \omega
$$

The differential phase parameter allows not only to obtain additional differentiated phase curves, but also to avoid the necessity to transmit the reference signal from the source dipole to the recording instrument. For the FDEMS method, apparent resistivity $(\rho)$ is calculated by dividing the signal $(\Delta \mathrm{U})$, normalized to the geometric coefficient $(\mathrm{k})$ at the observation site, by the amplitude of the control source current (I), according to the equation (2):

$$
\mathrm{P}=\mathrm{k} \times \Delta \mathrm{U} / \mathrm{I}
$$

Subjects of the interpretation in FDEMS are the amplitude apparent resistivity curves $(\varphi \omega)$, plotted on the log$\log$ chart and the curves of the differential phase parameter $(\Delta \varphi \omega)$, plotted on the semi-logarithmic charts (Fig.1).

\section{Features of the FDEMS curves above highly resistive} reference horizon

In order to solve various geological problems, it is necessary to map the surface of the high resistivity reference horizon occurring under the layers of overlying sediments and disintegrated low resistivity portion of the weathered bedrock. This problem is relevant for the exploration of mineral resources concentrated in the weathered bedrocks (bauxite, kaolin, and others), as well as for the geological engineering surveys in construction industry. The simplest solution model for this problem is a two layer medium with high resistivity reference horizon and the overlying low resistivity horizon, which can be either homogeneous or consist of several low resistivity layers. Figure 1 shows the resulting two layer amplitude $(\rho)$ and phase $(\Delta \varphi)$ curves EM field's electrical and magnetic components acquired in the equatorial layout.
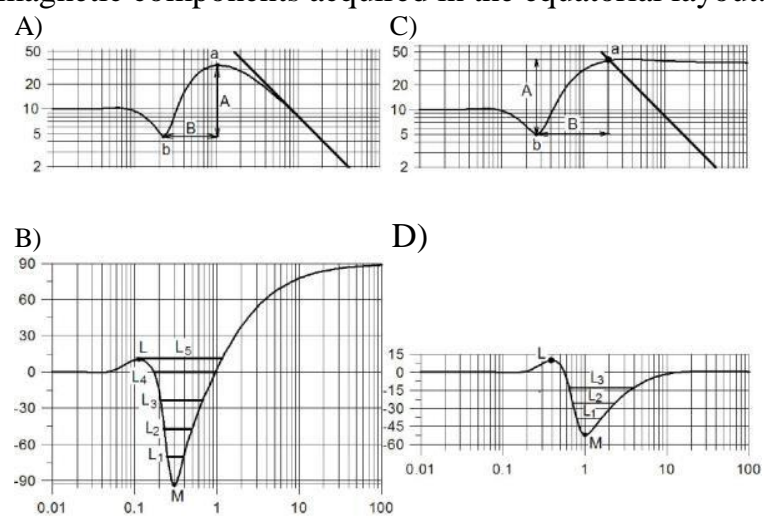

Fig.1 Amplitude and phase FDEMS curves of the vertical magnetic component $(\partial \mathrm{B} / \partial \omega)(\mathrm{A}, \mathrm{C})$ and the horizontal electric component $(\mathrm{Ex})(\mathrm{B}$, D) above the two-layer section with highly resistive second horizon.

The ratio between the soundings spacing $(\mathrm{R})$ and the depth of the reference horizon $(\mathrm{H})$ is 4 . For the cross-section with the high resistivity second layer, the amplitude curves show the horizontal asymptote which represents the resistance of the first layer, followed by a flat maximum, descending branch, distinct minimum, steep ascending branch and a maximum. In this case, the maximum of the magnetic component is very clear, and it is followed by the descending branch, which transfers to the asymptote of the nearest zone. The position of the asymptote is defined by the equation (3) and it is proportional only to the soundings spacing $(\mathrm{R})$ and is not influenced by the geoelectrical cross- section properties:

$$
\mathrm{P} \omega=1.3 \times \mathrm{R} \times \mathrm{f}
$$

In contrast to the magnetic component, the electric component has a very flat maximum, followed by very slow transition into the horizontal asymptote. The ordinate of the right asymptote uniquely defines the total longitudinal conductivity $(\mathrm{S})$ of low resistivity rocks overlying the high resistivity reference horizon (4):

$$
\mathrm{S}=\mathrm{R} / \rho \text { as }
$$

Where $\mathrm{R}$, is the distance between the transmitter and receiver dipoles, and $\rho$ as - as the value of the right horizontal asymptote ordinate.

In practice, instead of $\rho$ as it is more convenient to use the ordinate of the intersection point between the amplitude curve of the electric component and the asymptote of the nearest zone $\rho_{\mathrm{a}}$ (Fig.1).

The FDEMS method is the only electroprospecting technique where curves encompass direct information about the depth of the reference horizon. This information is expressed by the ratio between the soundings spacing $(\mathrm{R})$ and the depth of the reference horizon $(\mathrm{H})$, which is closely related to $\mathrm{A}, \mathrm{B}$ and $\mathrm{C}$ parameters of the amplitude curves. Parameters $\mathrm{A}$ and $\mathrm{B}$ are shown in Figure 1, while parameter $\mathrm{C}$ is determined from the equation $(5)^{[10]}$ :

$$
\mathrm{C}=\rho \min / \mathrm{R} 2 \times \mathrm{fmin}
$$

On the phase curves (Fig.1), the ordinate of the minimum $\Delta \varphi \min$, as well as the width of the minimum at the certain distance from its extreme point are related to the $\mathrm{R} / \mathrm{H}$ ratio ${ }^{[9]}$. In the former USSR, the FDEMS method was extensively used for purposes of mapping the crystalline basement's surface of the ancient shields and their slopes. In this paper we analyze some examples of such works.

\section{PRACTICAL APPLICATION OF THE FDEMS METHOD IN TOMAKOVSKYA AREA OF UKRAINIAN SHIELD}

The survey area was located in the central part of the Ukrainian shield in the Dnepropetrovsk region. Field work was carried out in 1978-1979 with NCHZ - 73 equipment (frequency range $10000-70 \mathrm{~Hz}$ ) and 100 and 200m soundings spacing (R). The average calculation error of $\rho \omega$ was $4.7 \%$. Instruments which were used for this survey did not have phase measurements capabilities. The geological objective of the survey was to map the surface of the crystalline bedrock, covered with a thick crust of weathered rocks and quaternary sediments. The total power of the low resistivity sediments was ranging from 10 to $100 \mathrm{~m}$. The type of the low resistivity crosssection was varying from two to four layered (KH). The typical FDEMS amplitude curves for 100 and $200 \mathrm{~m}$ soundings spacing (R) are shown in Figure 2. The application of express data interpretation methods using two layer dependencies for A, 
B, C parameters resulted in about $10 \%$ average error determining the depth of the crystalline rocks. Interpretation of the data sampled from 43 wells using the statistical method further reduced the error to $8 \%$. Separation of the training set into three classes: two-layer, three-layer type $\mathrm{H}$ and four-layer type $\mathrm{KH}$, produced the average error of $6 \%$, which is very close to the error in the field data. It was established, that the reduction of the average error determining $\mathrm{H}$ could be achieved by measuring two EM field components (horizontal electric and vertical magnetic) and using two soundings spacing's $(\mathrm{R}=100$ and $200 \mathrm{~m})$.
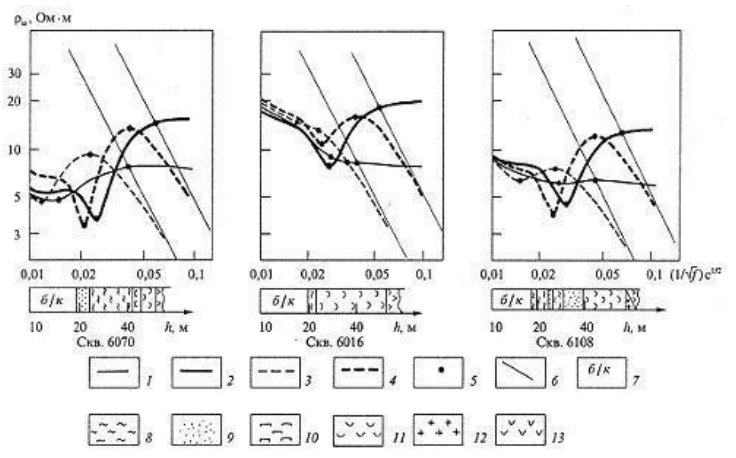

Fig.2 Types of FDEMS curves in the Tomakovskya area. From left to right: A- well \#6170, type A; B-well \#6016, type H, C-well\#6108, type HA; 1 Ex, $R=100 \mathrm{~m} ; 2$. Ex, $\mathrm{R}=200 \mathrm{~m}, 3 . \partial \mathrm{Bz} / \partial \omega, \mathrm{R}=100 \mathrm{~m}, 4$. $\partial \mathrm{Bz} / \partial \omega, \mathrm{R}=200 \mathrm{~m}$; peculiar points at the resistivity curves, asymptote of nearest zone; 7-interval of drilling without core; 8- shale, 9- sand; 10- kaolin; 11- weathering crust (mainly kaolin); 12-granites; 13 - amphibolites.

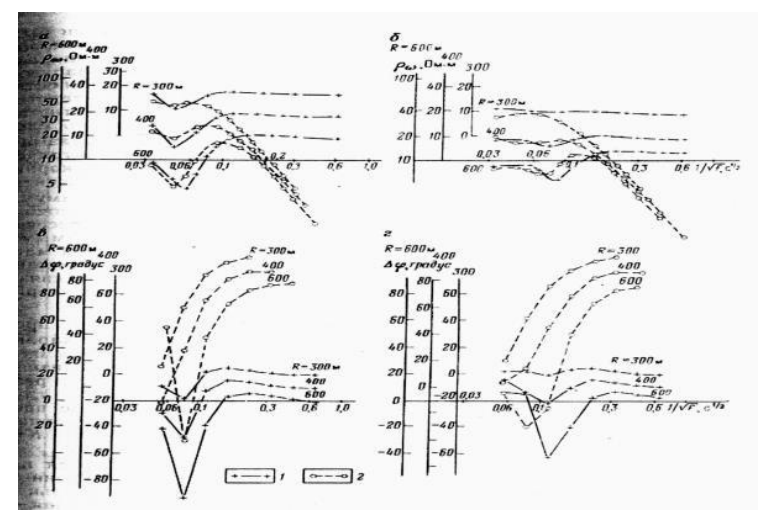

Fig. $3 \rho_{\omega}$ and $\Delta \varphi_{\omega}$ FDEMS curves obtained as results of the horizontal electric (1) and vertical magnetic field (2) measurements with EVA-203 equipment near the boreholes located at different depths from the crystalline basement (left - 130m; right - 219m).

\section{V.PRACTICAL APPLICATION OF THE FDEMS METHOD IN DNEPROVSKAYA AREA OF UKRAINIAN SHIELD}

The survey area was located at the south slope of the Ukrainian shield in the Zoporozhye region and, slightly south from the Dniepr River. The depth of the crystalline basement ranged from 60 to $300 \mathrm{~m}$. Along with the amplitude measurements FDEMS field survey in the Dneprovskaya area was performed using EVA-203 and NCHZ-73 equipment. Soundings were done with $\mathrm{R}=200$, 300 and $400 \mathrm{~m}$. The, the EVA equipment was also capable to carry out measurements of the differential phase parameter $(\Delta \varphi \omega)$. Examples of the amplitude and phase curves for three spacing's $(\mathrm{R}=300,400$ and $600 \mathrm{~m})$ are shown in Figure 3. The average field data achieved:

$\rho \omega=2.1 \%, \Delta \varphi \omega=1.5 \%$.

The differential phase curves $(\Delta \varphi \omega)$ proved to be very useful during the interpretation process as well and combination of amplitude and phase curves of two EM field components and soundings with three spacing's reduced the average error of mapping the surface of the bedrock to $2 \%$.

\section{CONCLUSIONS}

Effective combination of geometric and induction soundings techniques in the FDEMS method allows to map the boundaries with the exceptional accuracy.

Contributing factors to the improved accuracy of mapping the boundaries with FDEMS are:

Implementation of the amplitude and phase measurements;

Measurements of at least two electromagnetic field components (Ex, Bz);

Carrying out soundings with several separations (R).Utilizing modern high precision data acquisition system based on the multifunction multichannel wideband EM receivers with built in hardware capabilities for the full realization of all FDEMS method advantages in the field.

It is apparent, that the precision of mapping the boundaries can be greatly improved by exploiting technological advancements of modern high precision wideband multifunction EM receivers such as GEPARD-4 and GEPARD8.The FDEMS method at the present time should be considered as one of the most effective electroprospecting techniques for geological engineering surveys, groundwater prospecting and mining exploration.

\section{REFERENCE}

[1] McNeill, J.D. 1980. Electromagnetic Terrain Conductivity Measurements at Low Induction Numbers. Geonics Ltd., Technical Note TN-6, 15 pgs.

[2] Kaufman, A.A., Keller, G.V., 1983, Frequency and Transient Soundings, Elsevier, New York, New York, 685 pgs.

[3] Zonge, K.L. and Hughes, L.J., 1991, Controlled source audiofrequency Magnetotellurics. In Nabighian, M.N. (Ed.), Electromagnetic Methods in Applied Geophysics, Volume 2, Applications: Parts A and Part B, Society of Exploration Geophysicists Investigations in Geophysics 3, 713-809, Doi: 10.1190/1.9781560802686.ch9

[4] Vanyan L.L., 1965, The bases of electromagnetic soundings, Moscow, "Nedra", 105 pgs.

[5] Ivanov, A.P., 1983, Frequency Electromagnetic Sounding, Academy of Science of USSR, Institute Physics of the Earth, $227 \mathrm{pgs}$.

[6] Gorunov A.S., Ingerov A.I., Kulikov A.V., 1987, Equipment EVA-203 application for Frequency Electromagnetic sounding, Application Geophysics, 117, 62-69.

[7] Ingerov A.I., Soldatenko, V.P., 1998, About accuracy of the depth of high resistive reference horizon estimation by methods of frequency electromagnetic sounding, The reports of National Academy of Science of Ukraine, 12, 123-128.

[8] Ingerov, I., 2011, Method of multifrequency magnetovariational profiling (MVP), EMS-2011, St. Petersburg, Russia, Abstracts, Vol.2, 449-454.

[9] Kulikov A.V., Shemyakin E.A, 1978, Electroprospecting by Phase Method of Induced Polarization, Moscow, "Nedra", 248 pgs. 